\title{
Feedback Stabilization of Nonlinear Stochastic Time-Delay Systems With State and Control-Dependent Noise
}

\author{
Weihai Zhang, Bor-Sen Chen and Qinghua Li
}

\begin{abstract}
This paper mainly treats with the feedback stabilization of nonlinear stochastic time-delay systems with state and control-dependent noise, some locally/globally robustly stabilizable conditions are correspondingly given independent of delay length. Several previous consequences are extended to more general systems with both state and control-dependent noise.
\end{abstract}

\section{INTRODUCTION}

The stabilizability of linear stochastic control systems governed by Itô differential equations has been investigated by many authors, see [7], [8], [10] and the references therein. In recent years, the stabilization of nonlinear stochastic systems has also become a popular topic and attracted a great deal of attentions, see [4]-[6],etc.. This paper is on robust stabalization of nonlinear stochastic systems with uncertain time-delay. So far, most work related to such problems are focused on the deterministic timedelay systems, there are only a few authors treating with the stability and stabilization of the nonlinear stochastic delay systems ( [1], [2], [13], [14], etc.), especially, most of the criteria given are based on matrix norm , matrix measure operation or ARE, which makes these results more conservative and inconvenient to be used in some cases.

Based on [11], in this paper, we mainly use linear matrix inequality (LMI)-based approach to treat with the stabilization of more general nonlinear stochastic systems with both state and control-dependent noise, which makes our results more applicable and less conservative. In Section II, some preliminary work is presented; Section III is on the robust stabilization of nonlinear stochastic time-delay systems, some useful criteria are presented in terms of LMIs independent of the length of delay; Section IV concludes this note by some remarks.

\section{PROBLEM SETTING}

For convenience, we adopt the following notations:

$\mathcal{S}_{n}$ : the set of all real $n \times n$ symmetric matrices;

$A^{\prime}$ : the transpose of the corresponding matrix $A$;

$A \geq 0(A>0):$ positive semidefinite (positive definite) matrix $A$;

W. Zhang is with the College of Mechanical and Electrical Engineering, Shandong Institute of Light Industry, Jinan 250100, China Email: tgzzwh@hotmail.com

B.S.Chen is with the Department of Electrical Engineering, National Tsing Hua University, Hsinchu 30043, Taiwan Email: bschen@moti.ee.nthu.edu.tw

Q. Li is with the College of Mechanical and Electrical Engineering, Shandong Institute of Light Industry, Jinan 250100, China Email: lqhlxzesohu.com
$I$ : identity matrix.

$C_{2}^{0}(\{t>0\} \times U)$ : class of functions $V(t, x)$ twice continuously differential with respect to $x \in U$ and once continuously differential with respect to $t>0$, except possibly at the point $x=0$.

Consider the following stochastic controlled system governed by an Itô differential equation

$$
d x(t)=f(x(t), u(t)) d t+\sigma(x(t), u(t)) d w(t)
$$

In the above, $x(t) \in \mathcal{R}^{n}$ is called the system state, $u(t) \in$ $\mathcal{R}^{m}$ the control input, and $w(t)$ is the standard Wiener process defined on the probability space $(\Omega, \mathcal{F}, \mathcal{P})$, with natural filter $\mathcal{F}_{t}$ generated by $w(\cdot)$ up to time $t$. Without loss of generality, we can suppose $w(t)$ is one-dimensional. Assume $u(t)$ is an adapted and measurable process with respect to $\mathcal{F}_{t}, f(0, u) \equiv 0, \sigma(0, u) \equiv 0$, i.e., $x \equiv 0$ is an equilibrium point of (1). Under very general conditions on $f, \sigma$ and $u(t)$, stochastic controlled system (1) has a unique strong solution $x_{s, \xi}(t)$ for any $t \geq s \geq 0$, and initial state $x(s)=\xi \in \mathcal{R}^{n}$ [3]. We first introduce the following definition:

Definition 1. We say that system (1) is locally asymptotically stabilizable via linear state feedback $\mathrm{u}(\mathrm{t})=\mathrm{Kx}(\mathrm{t})$, if the trivial solution $x \equiv 0$ of the closed-loop system

$$
d x(t)=f(x(t), K x(t)) d t+\sigma(x(t), K x(t)) d w(t)
$$

is asymptotically stable in probability(see, e.g.,[6]), i.e., for any $s \geq 0$ and $\varepsilon>0$

$$
\lim _{\xi \rightarrow 0} \mathcal{P}\left(\sup _{t \geq s}\left\|x_{s, \xi}(t)\right\|>\varepsilon\right)=0
$$

and

$$
\lim _{\xi \rightarrow 0} \mathcal{P}\left(\lim _{t \rightarrow \infty} x_{s, \xi}(t)=0\right)=1
$$

where $K$ is a suitable dimensional constant matrix. In addition, if the solution $x \equiv 0$ of the closed-loop system (2) is asymptotically stable in the large (see, e.g.,[6]), i.e., if (3) and

$$
\mathcal{P}\left(\lim _{t \rightarrow \infty} x_{s, \xi}(t)=0\right)=1
$$

hold, then we say that (1) is globally asymptotically stabilizable via linear state feedback $\mathrm{u}(\mathrm{t})=\mathrm{Kx}(\mathrm{t})$.

Remark 1. It is well known [6] that if there exists a neighborhood $U$ of the origin, a Lyapunov function $V(t, x) \in C_{2}^{0}(\{t>0\} \times U), V(t, x)>0$ in domain $\{t>0\} \times U$, which has infinitesimal upper limit, i.e.,

$$
\lim _{x \rightarrow 0} \sup _{t>0} V(t, x)=0
$$


satisfying

$$
\begin{aligned}
\mathcal{L} V(t, x)= & \frac{\partial V(t, x)}{\partial t}+f^{\prime}(x, K x) \frac{\partial V(t, x)}{\partial x} \\
& +\frac{1}{2} \sigma^{\prime}(x(t), K x(t)) \frac{\partial^{2} V(t, x)}{\partial x^{2}} \sigma(x(t), K x(t)) \\
& <0
\end{aligned}
$$

for $x \neq 0$, then $x(t) \equiv 0$ of system (2) is asymptotically stable in probability. Furthermore, if $V(t, x)$ also admits the following property

$$
\inf _{t>0} V(t, x) \rightarrow \infty, \text { as }\|x\| \rightarrow \infty
$$

and $U$ is replaced by the total space $\mathcal{R}^{n}$, then $x(t) \equiv 0$ of system (2) is asymptotically stable in the large. Where $\mathcal{L}$ is so- called the infinitesimal generator of (2).

Now, we suppose that the linear parts of $f(x, u)$ and $\sigma(x, u)$ can be separated out as follows:

$$
\begin{aligned}
& f(x, u)=A x+B u+H_{0}(x, u), H_{0}(0, u) \equiv 0, \\
& \sigma(x, u)=C x+D u+H_{1}(x, u), H_{1}(0, u) \equiv 0
\end{aligned}
$$

then the nonlinear stochastic system (1) can be rewritten as $d x=\left(A x+B u+H_{0}(x, u)\right) d t+\left(C x+D u+H_{1}(x, u)\right) d w$

where $A, B, C$ and $D$ are constant matrices. Below, we will discuss the stabilization of system (8).

\section{FEEDBACK STABILIZATION OF STOCHASTIC TIME-DELAY SYSTEMS}

In this section, we investigate the robust stabilization of the following stochastic time-delay system governed by Itô differential equations of the form

$$
\left\{\begin{aligned}
& d x(t)=(A x(t)+B x(t-\tau)+B_{1} u(t) \\
&+\left.H_{0}(x(t), x(t-\tau), u(t))\right) d t \\
&+\left(C x(t)+D x(t-\tau)+D_{1} u(t)\right. \\
&\left.+H_{1}(x(t), x(t-\tau), u(t))\right) d w \\
& x(t)=\phi(t) \in L^{2}\left(\Omega, \mathcal{F}_{0}, C\left([-\tau, 0], \mathcal{R}^{n}\right)\right), t \in[-\tau, 0]
\end{aligned}\right.
$$

where $A, B, B_{1} C, D$ and $D_{1}$ are constant matrices, $\tau>0$ is an uncertain time delay, $H_{i}(0, \cdot, \cdot) \equiv 0, i=0,1$. Under very mild conditions on $H_{i}(\cdot, \cdot, \cdot), i=0,1$, (9) exists a unique global solution [13]. We will prove that, for some special cases of the above system, robust stability and stabilization can be given via LMIs, which is very efficient for practical computation.

Definition 2. Stochastic time delay differential system (9) is called locally robustly stabilizable if there exists a constant state feedback control law $u(t)=u(x(t))=$ $K x(t)$, such that the equilibrium point of the closed-loop system

$$
\left\{\begin{aligned}
d x(t)= & \left(\left(A+B_{1} K\right) x(t)+B x(t-\tau)\right. \\
& \left.+H_{0}(x(t), x(t-\tau), K x(t))\right) d t \\
& +\left(\left(C+D_{1} K\right) x(t)+D x(t-\tau)\right. \\
& \left.+H_{1}(x(t), x(t-\tau), K x(t))\right) d w \\
x(t)= & \phi(t) \in L^{2}\left(\Omega, \mathcal{F}_{0}, C\left([-\tau, 0], \mathcal{R}^{n}\right)\right), t \in[-\tau, 0]
\end{aligned}\right.
$$

is asymptotically stable in probability for all $\tau \geq 0$. It is called globally robustly stabilizable, if (10) is robustly stable [1], i.e., the equilibrium point of (10) is asymptotically stable in the large for all $\tau \geq 0$.

A very general theorem is as follows.

Theorem 1. If the following matrix inequality

$$
Z:=\left[\begin{array}{l}
\left\{P\left(A+B_{1} K\right)+\left(A+B_{1} K\right)^{\prime} P+Q\right. \\
\left.+\left(C+D_{1} K\right)^{\prime} P\left(C+D_{1} K\right)\right\} \\
B^{\prime} P+D^{\prime} P\left(C+D_{1} K\right) \\
P B+\left(C+D_{1} K\right)^{\prime} P D \\
D^{\prime} P D-Q
\end{array}\right]<0
$$

has solutions $P>0, Q>0$ and $K \in \mathcal{R}^{m \times n}$, and

$$
\lim _{\|x\| \rightarrow 0} \sup _{y \in \mathcal{R}^{n}}\left\|H_{i}(x, y, K x)\right\| /\|x\|=0, i=0,1
$$

then system (9) can be locally robustly stabilized by $u(t)=$ $K x(t)$.

To prove Theorem 1, we construct the LyapunovKrasovskii functional ([9]) as follows:

$$
V(t, x)=x^{\prime}(t) P x(t)+\int_{0}^{\tau} x^{\prime}(t-s) Q x(t-s) d s
$$

where $P>0$ and $Q>0$ are the solutions of (11). Letting $\mathcal{L}_{1}$ be the infinitesimal generator of the closed-loop system (10), where $K$ is a solution to (11), then we have

$$
\begin{aligned}
\mathcal{L}_{1} V(t, x(t))= & \left(\left(C+D_{1} K\right) x(t)+D x(t-\tau)\right. \\
& \left.+H_{1}(x(t), x(t-\tau), K x(t))\right)^{\prime} P \\
& \cdot\left(\left(C+D_{1} K\right) x(t)+D x(t-\tau)\right. \\
& +H_{1}(x(t), x(t-\tau), K x(t)) \\
& +2<\left(A+B_{1} K\right) x(t)+B x(t-\tau) \\
& +H_{0}(x(t), x(t-\tau), K x(t)), P x(t)> \\
& +x^{\prime}(t) Q x(t)-x^{\prime}(t-\tau) Q x(t-\tau)
\end{aligned}
$$

Rearranging (13) yields

$$
\begin{aligned}
& \mathcal{L}_{1} V(t, x(t))=x^{\prime}(t)\left(P\left(A+B_{1} K\right)\right. \\
& +\left(A+B_{1} K\right)^{\prime} P \\
& \left.+Q+\left(C+D_{1} K\right)^{\prime} P\left(C+D_{1} K\right)\right) x(t) \\
& +2 x^{\prime}(t)\left(P B+\left(C+D_{1} K\right)^{\prime} P D\right) x(t-\tau) \\
& +x^{\prime}(t-\tau)\left(D^{\prime} P D-Q\right) x(t-\tau) \\
& +2 H_{0}^{\prime}(x(t), x(t-\tau), K x(t)) P x(t) \\
& +2 H_{1}^{\prime}(x(t), x(t-\tau), K x(t)) P D x(t-\tau) \\
& +2 H_{1}^{\prime}(x(t), x(t-\tau), K x(t)) P\left(C+D_{1} K\right) x(t) \\
& +H_{1}^{\prime}(x(t), x(t-\tau), K x(t)) P H_{1}(\cdot, \cdot, \cdot) \\
= & \quad x(t)]^{\prime} Z[x(t)] \\
& x(t-\tau)] \\
+ & 2 H_{0}^{\prime}(x(t), x(t-\tau), K x(t)) P x \\
+ & 2 H_{1}^{\prime}(x(t), x(t-\tau), K x(t)) P\left(C+D_{1} K\right) x(t) \\
+ & 2 H_{1}^{\prime}(x(t), x(t-\tau), K x(t)) P D x(t-\tau)
\end{aligned}
$$




$$
+H_{1}^{\prime}(x(t), x(t-\tau), K x(t)) P H_{1}(\cdot, \cdot, \cdot)
$$

Then by (11), $Z<0$, so

$$
\begin{aligned}
\left(x^{\prime}(t), x^{\prime}(t-\tau)\right) Z\left[\begin{array}{c}
x(t) \\
x(t-\tau)
\end{array}\right] \leq & \lambda_{\max }(Z)\left(\|x(t)\|^{2}\right. \\
& \left.+\|x(t-\tau)\|^{2}\right)
\end{aligned}
$$

In addition, by (12), for any $\epsilon>0$, there exists $\delta>0$, such that when $\|x\|<\delta$,

$\left\|H_{0}(x, y, K x)\right\| \leq \epsilon\|x\|,\left\|H_{1}(x, y, K x)\right\| \leq \epsilon\|x\|, \forall y \in \mathcal{R}^{n}$

Hence

$$
\begin{aligned}
& 2 H_{0}^{\prime}(x(t), x(t-\tau), K x(t)) P x(t) \\
& +2 H_{1}^{\prime}(x(t), x(t-\tau), K x(t)) P C x(t) \\
& +2 H_{1}^{\prime}(x(t), x(t-\tau), K x(t)) P D x(t-\tau) \\
& +H_{1}^{\prime}(\cdot, \cdot, \cdot) P H_{1}(\cdot, \cdot, \cdot) \\
\leq & 2 \epsilon\|C\| \cdot\|P\| \cdot\|x(t)\|^{2} \\
& +2 \epsilon\|D\| \cdot\|P\| \cdot\|x(t)\| \cdot\|x(t-\tau)\| \\
& \left.+\epsilon^{2}\|P\| \cdot\|x(t)\|^{2}+2 \epsilon\|P\| \cdot\|x(t)\|^{2}\right)
\end{aligned}
$$

By inequality $|a b| \leq \frac{1}{2}\left(a^{2}+b^{2}\right)$, from (16) follows

$$
\begin{aligned}
& 2 H_{0}^{\prime}(x(t), x(t-\tau), K x(t)) P x \\
& +2 H_{1}^{\prime}(x(t), x(t-\tau), K x(t)) P C x(t) \\
& +2 H_{1}^{\prime}(x(t), x(t-\tau), K x(t)) P D x(t-\tau) \\
& +H_{1}^{\prime}(\cdot, \cdot, \cdot) P H_{1}(\cdot, \cdot, \cdot \cdot) \\
\leq & \left(2 \epsilon\|C\|+\epsilon\|D\|+2 \epsilon+\epsilon^{2}\right)\|P\| \cdot\|x(t)\|^{2} \\
& +\epsilon\|D\|\|P\| \cdot\|x(t-\tau)\|^{2} \\
\leq & -\frac{1}{2} \lambda_{\max }(Z)\left(\|x\|^{2}+\|x(t-\tau)\|^{2}\right.
\end{aligned}
$$

if we take $\epsilon$ sufficiently small, such that

$$
\max \left(2 \epsilon\|C\|+\epsilon\|D\|+2 \epsilon+\epsilon^{2}, \epsilon\|D\|\right) \leq-\frac{\lambda_{\max }(Z)}{2\|P\|}
$$

then from (15) together with (17) follows

$$
\mathcal{L}_{1} V(t, x(t)) \leq \frac{1}{2} \lambda_{\max }(Z)\left(\|x(t)\|^{2}+\|x(t-\tau)\|^{2}\right)<0
$$

That is, $\mathcal{L}_{1} V(t, x(t))<0$ in the domain $\{t>0\} \times U:=$ $\{t>0\} \times\{x:\|x\|<\delta\}$ for $x \neq 0$. In addition, note that $V(t, x)$ can be written as

$$
V(t, x(t))=x^{\prime}(t) P x(t)+\int_{t-\tau}^{t} x^{\prime}(s) Q x(s) d s
$$

hence, in the domain $\{t>0\} \times U$, (6) holds. This ends the proof of Theorem 1.

However, since (11) is not an LMI on variables $P, Q$ and $K$, it is not convenient to use Theorem 1 in practice. However, from Theorem 1, we can derive some useful results, which can be expressed in terms of LMIs.

Corollary 1. Under the conditions of Theorem 1 , if $H_{i} \equiv$ $0, i=0,1$, then the linear stochastic time-delay system

$$
\begin{aligned}
d x(t)= & \left(A x(t)+B x(t-\tau)+B_{1} u(t)\right) d t \\
& +\left(C x(t)+D x(t-\tau)+D_{1} u(t)\right) d w
\end{aligned}
$$

is globally robustly stabilizable. Especially, if $D=0$, and the following LMI

$$
\begin{aligned}
& {\left[\begin{array}{l}
A \hat{P}+\hat{P} A^{\prime}+B_{1} Y+Y^{\prime} B_{1}^{\prime}+B \hat{Q} B^{\prime} \\
C \hat{P}+D_{1} Y \\
\hat{P}
\end{array}\right.} \\
& \left.\begin{array}{rr}
\hat{P} C^{\prime}+Y^{\prime} D_{1}^{\prime} & \hat{P} \\
-\hat{P} & 0 \\
0 & -\hat{Q}
\end{array}\right]<0
\end{aligned}
$$

admits solutions $\hat{P}>0, \hat{Q}>0$ and $Y \in \mathcal{R}^{m \times n}$, then

$$
d x=\left(A x+B x(t-\tau)+B_{1} u\right) d t+\left(C x+D_{1} u\right) d w
$$

is globally robustly stabilizable. In this case, the stabilizing feedback control law $u(t)=K x(t)=Y \hat{P}^{-1} x(t)$.

Proof. If $H_{0}(\cdot, \cdot, \cdot) \equiv 0, H_{1}(\cdot, \cdot, \cdot) \equiv 0$, then $\mathcal{L}_{1} V(t, x(t))<0$ for $(t, x) \in\{t>0\} \times \mathcal{R}^{n}$, except possibly at $x=0$, and (7) clearly holds. Thus, the first part of Corollary 1 is proved.

Furthermore, if $D=0,(11)$ degenerates into

$$
\left[\begin{array}{cc}
\left\{P\left(A+B_{1} K\right)+\left(A+B_{1} K\right)^{\prime} P+Q\right. & P B \\
\left.+\left(C+D_{1} K\right)^{\prime} P\left(C+D_{1} K\right)\right\} & \\
B^{\prime} P & -Q
\end{array}\right]<0
$$

which by Schur's complement is equivalent to

$$
\begin{aligned}
P\left(A+B_{1} K\right)+ & \left(A+B_{1} K\right)^{\prime} P+P B Q^{-1} B^{\prime} P+Q \\
+ & +\left(C+D_{1} K\right)^{\prime} P\left(C+D_{1} K\right)<0
\end{aligned}
$$

Then pre- and postmultiplying (21) by $P^{-1}$, we have

$$
\begin{aligned}
& \left(A+B_{1} K\right) P^{-1}+P^{-1}\left(A+B_{1} K\right)^{\prime}+B Q^{-1} B^{\prime}+P^{-1} \\
& \cdot\left(C+D_{1} K\right)^{\prime} P\left(C+D_{1} K\right) P^{-1}+P^{-1} Q P^{-1}<0
\end{aligned}
$$

Setting $\hat{P}:=P^{-1}, Y=: K P^{-1}=K \hat{P}$, and $\hat{Q}:=Q^{-1}$; again, by Schur's complement, (23) is equivalent to (19). Thus the second part of Corollary 1 is also proved. 
When $D=D_{1}=H_{0}=H_{1} \equiv 0$, the analogous problem to Corollary 1 is also considered by [5] via delay feedback, where the main result is expressed by means of a algebraic Riccati-type equation.

The following result is an immediate corollary of Theorem 1.

Corollary 2. The unforced system

$$
\begin{aligned}
d x(t)= & (A x(t)+B x(t-\tau)) d t \\
& +(C x(t)+D x(t-\tau)) d w
\end{aligned}
$$

is robustly stable, if the following LMI

$$
\left[\begin{array}{cc}
P A+A^{\prime} P+C^{\prime} P C+Q & P B+C^{\prime} P D \\
B^{\prime} P+D^{\prime} P C & D^{\prime} P D-Q
\end{array}\right]<0
$$

has solutions $P>0, Q>0$.

Another description for robust stability of (24) was given in [1] via the existence of positive solutions of the algebraic Riccati-type equation

$$
\begin{aligned}
P A+ & A^{\prime} P+W+C^{\prime} P C+D^{\prime} P D \\
& +\left(P B+C^{\prime} P D\right) W^{-1}\left(P B+C^{\prime} P D\right)+R \\
& =0
\end{aligned}
$$

One can easily show that Corollary 2 is equivalent to (i) of Theorem 1 in [1].

Corollary 3. The stochastic linear time-delay system

$$
\begin{aligned}
d x(t)= & \left(A x(t)+B x(t-\tau)+B_{1} u(t)\right) d t \\
& +(C x(t)+D x(t-\tau)) d w
\end{aligned}
$$

is globally robustly stabilizable, if the following LMI

$$
\left[\begin{array}{ccc}
P A+A^{\prime} P+C^{\prime} P C+Q & \sqrt{2} P B_{1} & P B+C^{\prime} P D \\
\sqrt{2} B_{1}^{\prime} P & -Q & 0 \\
B^{\prime} P+D^{\prime} P C & 0 & D^{\prime} P D-Q
\end{array}\right]
$$

$$
<0
$$

admits solutions $P>0$ and $Q>0$. Moreover, the stabilizing feedback control law $u(t)=Q^{-1} B_{1}^{\prime} P x(t)$.

Proof. Applying Theorem 1, this corollary is easily obtained.

Remark 2. All results obtained in this section can be extended without difficulty to systems with multiple delays and independent stochastic perturbations .

\section{CONCLUSIONS}

In the above sections, we have discussed the static statefeedback stabilization of nonlinear stochastic systems with state and control-dependent noise. Theorem 1 is on the locally robust stabilization of nonlinear stochastic timedelay systems, from which we derive some useful results for globally robust stabilization (stability) of the corresponding linearizable systems. All consequences except Theorem 1 can be expressed in terms of LMIs, which makes them more practically applicable [7].

\section{ACKNOWLEDGMENTS}

The authors gratefully acknowledge the reviewers' comments.

\section{REFERENCES}

[1] E.I.Verriest and P. Florchinger, Stability of stochastic systems with uncertain time delays, Systems and Control Lett., vol. 24, 1995, pp 41-47.

[2] E.I.Verriest and M.K.H. Fan, "Robust Stability of Nonlinearly Perturbed Delay Systems", in 35th IEEE Conference on Decision and Control, Kobe, Japan , 1996, pp. 2090-2091.

[3] L. Arnold, Stochastic differential equations: Theory and applications, Wiley, New York; 1974.

[4] P. Florchinger, Feedback stabilization of affine in the control stochastic differential systems by the control Lyapunov function method, SIAM J.Contr.Optim., vol. 35, 1997, pp 500-511.

[5] P. Florchinger and E. I. Verriest, "Stabilization of nonlinear stochastic systems with delay feedback", in 32nd IEEE Conference on Decision and Control, San Antonio, TX, WM-12, 1993.

[6] R.Z. Has'minskii, Stochastic Stability of Differential Equations, Sijthoff and Noordhoff, Alphen a/d Rijn; 1980.

[7] S. Boyd, L. El Ghaoui, E. Feron and V. Balakrishnan, Linear matrix inequalities in system and control theory, SIAM, Philadelphia, PA: 1994

[8] U. G. Haussmann, Stability of linear systems with control dependent noise, SIAM J. Contr, vol. 11, 1973, pp.382-394.

[9] V.Kolmanovskii and A.Myshkis, Applied Theory of Functional Differential Equations, Kluwer Academic Publishers, Dordrecht; 1992.

[10] W. Zhang, A note on the stabilizability of the stochastic systems", Chinese Journal of Engineering Mathematics, vol. 17, 2000, pp 129132 .

[11] W. Zhang and B.S.Chen, Local stabilizability of non-linear stochastic systems with state and control-dependent noise, The 4th Asian Control Conference, Singapore, 2002.

[12] W. Zhang and B.S. Chen, On stabilizability and exact observability of stochastic systems with applications, Automatica, vol. 40, 2004, pp 87-94.

[13] X. Mao, Stochastic Differential Equations and Their Applications, Horwood Publication, Chichester; 1997.

[14] X. Mao, Robust stability of uncertain stochastic differential delay equations, Systems and Control Lett., vol. 35, 1998, pp 325-336. 1998

[15] Z. Y. Gao and N. U. Ahmed, Stabilizability of certain stochastic systems, Int. J. Systems Sci., vol. 17, 1986, pp 1175-1185.

[16] Z. Y. Gao and N. U. Ahmed, Feedback stabilizability of non-linear stochastic systems with state-dependent noise, Int. J. Contr., vol. 45, 1987, pp 729-737. 